\title{
Grain Size Measurements - Variables to Consider
}

\author{
Janice Klansky
}

Buehler Ltd., 41 Waukegan Rd. Lake Bluff, IL 60044

In order to better understand the influence of the welding process on the mechanical properties of a finished component, it is useful to examine a metallographic cross-section. A typical weld will display three distinct regions: the solidified weld metal, grain growth and recrystallization due to the temperature gradient introduced by the welding process. Each region can have a dramatically different grain size and anisotropy. The goal of this study was to examine some of the variables surrounding automatic image analysis grain size measurements and determine some recommended practices when working with specimens with irregular microstructures.

Standardized approaches to measurement techniques have evolved with the technology available. For example, comparison charts and tables had determined the magnification requirements whereas the newer automated imaging standards reference the number of features per field of view or a set calibration factor. In addition, automation introduces new questions such as: just how well detected do all of the boundaries have to be to get an accurate answer and what are the consequences of having too few or too many grains in the field of view.

Two types of specimens were examined in order to understand the measurement process. The first was a plain carbon steel specimen with an equiaxed structure (Figure 1). Images from this specimen were used as a standard since most grain size methods assume an anisotropy value less than three. The second was a cross-section of a weld (Figure 2). This provided a variety of grain sizes and varying degrees of anisotropy.

Images of the equiaxed structure were captured at a resolution of 3072 x 3840 pixels. The grain size was measured across the entire field of view based on a count of the grains (planimetric method) and $\mathrm{x}$ and $\mathrm{y}$ intercepts [1,2]. Next the incomplete grains on the image boundaries were removed and grain size was determined based on the individual grain areas [2]. This procedure was repeated on subsections of the image to determine the influence of the relative number of grains on the boundaries compared to the total number of grains present in the image. Figure 3 demonstrates that most of the methods show an increase in the grain size number when fewer than fifty grains are present. The exception was the planimetric approach using a rectangle, which displayed no noticeable change in the grain size number.

Two of the measurement methods were then employed on a weld cross-section. In addition to calculating grain size, the $\mathrm{x}$ and $\mathrm{y}$ centroids of the grains were tracked to determine the relative positions of the grains. Figure 4 displays the grain size results relative to position using a constant magnification across the weld zone. Where the results from the two methods diverge there are either less than 50 grains present in the field of view or more than 300 grains. The difference in the average grain size is further amplified by the calculation method for average grain size. When individual areas were used to determine grain size the average was weighted by the number of grains present, not the actual area represented by the grains. The planimetric approach is more representative of an area weighted average. 
[1] "Standard Test Methods for Determining Average Grain Size," E 112-96, Annual Book of ASTM Standards, ASTM, 2000.

[2] "Standard Test Methods for Determining Average Grain Size Using Semiautomatic and Automatic Image Analysis," E 1382-97, Annual Book of ASTM Standards, ASTM, 2000.

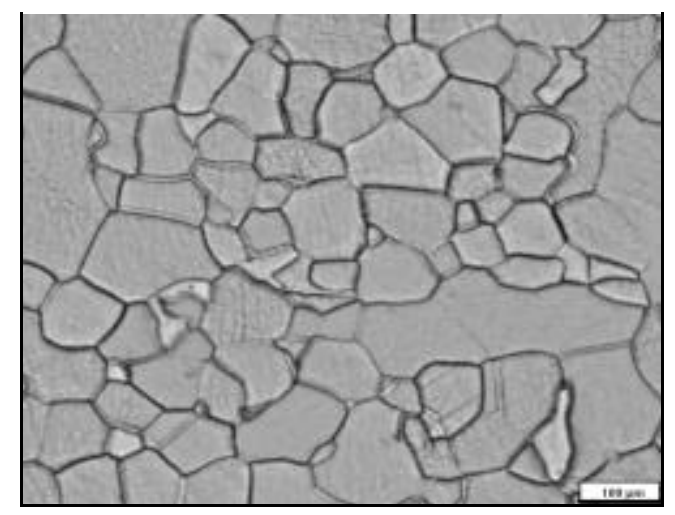

FIG. 1. A section of the equiaxed grain size image.

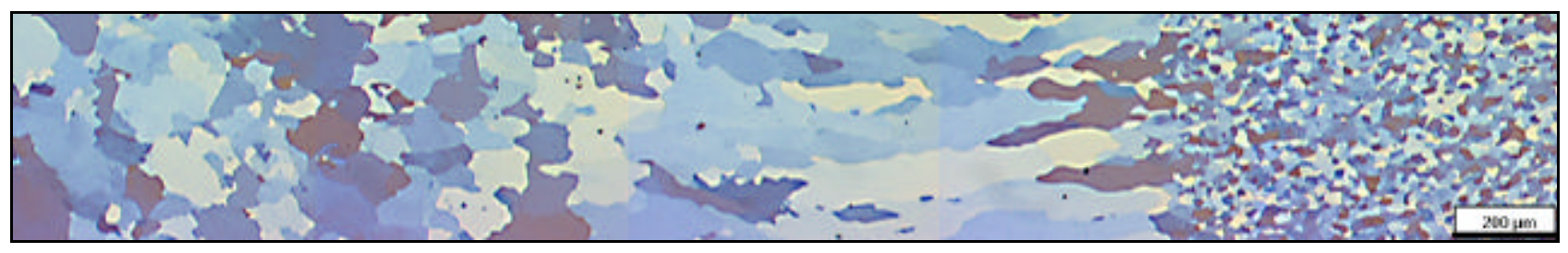

FIG. 2. A metallographic cross-section of a weld specimen.

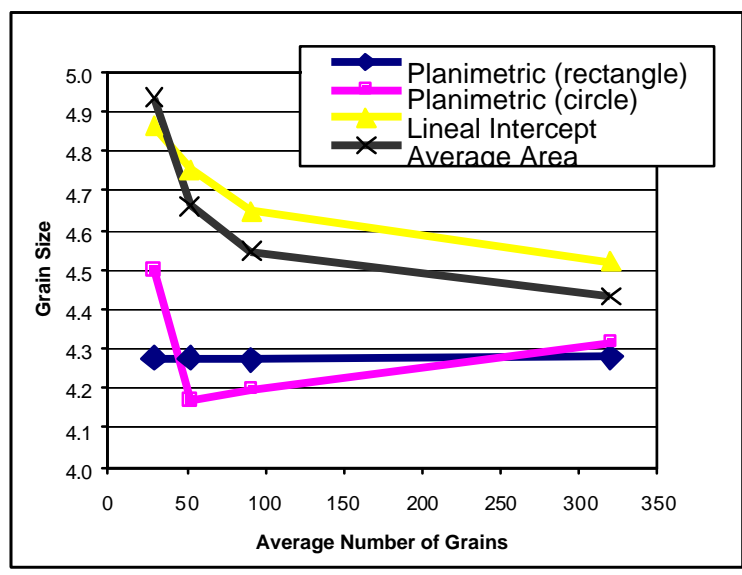

FIG. 3. A comparison of grain size measurement methods.

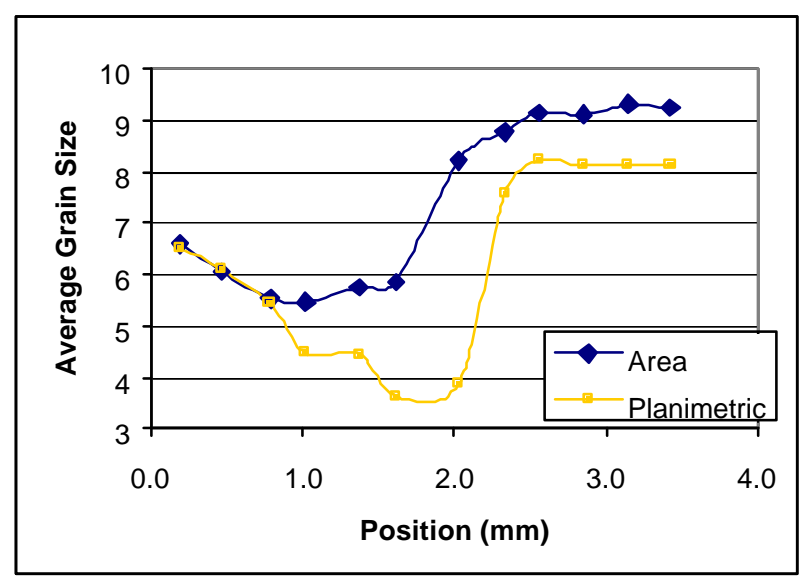

FIG. 4. A comparison of grain size measurement methods across a weld cross-section. 